\title{
applications \\ de la photo-interprétation \\ et de la télédétection \\ à la géologie de l'ingénieur
}

A. applications pratiques de la photo-interprétation et de la télédétection
par
D. Galmier
R. Lacot
R. Richard
Ingénieurs-Conseils

B. exemple d'utilisation de la photogéologie en cartographie géotechnique à petite échelle

par

R. Vyain, BRGM

C. problème posé par la fondation du barrage de l'Arnon (Cher)

par

J.-Y. Scanvic, BRGM

D. esquisse sismo-tectonique de Provence

par

G. Weecksteen, BRGM 


\section{RESUME}

Applications pratiques de la photo-interprétation et de la télédétection par D. Galmier, R. Lacot, R. Richard.

L'utilisation de la photo-interprétation permet de mieux définir les secteurs à urbaniser en repérant les anomalies de terrain. Elle donne en outre une bonne approche des problèmes hydrogéologiques, facilite la recherche des matériaux et oriente les études et reconnaissances de terrain à entreprendre.

Les nombreux exemples donnés montrent l'intérêt que présente cette approche des problèmes.

Exemple d'utilisation de la photogéologie en cartographie géotechnique à petite échelle par R. Vyain.

L'exemple cité met en évidence une utilisation de la photogéologie appliquée à la cartographie géotechnique en zone équatoriale. Elle a permis de reconnaître une grande surface et de localiser les zones géotechniques complexes nécessitant une étude plus approfondie.

Problème posé par la fondation du barrage de I'Arnon (Cher) par J.-Y. Scanvic.

La photo aérienne classique permet de déterminer les réseaux de fractures à l'échelon local sans préciser leur importance régionale. L'examen comparé avec des images satellites a montré l'existence d'une faille régionale passant sur le site du barrage, retrouvée pendant les travaux.

Esquisse sismo-tectonique de Provence à $1 / 250000$ par G. Weecksteen.

L'utilisation des images satellites et des photos aériennes a permis d'esquisser une carte sismotectonique de la Provence.

Des problèmes de méthodes se posent dans le traitement des informations.

\section{SUMMARY}

Practical Applications of Photointerpretation and Teledetection, by D. Galmier, R. Lacot and R. Richard.

By detecting unusual site features, photointerpretation permits more precise definition of areas for urban development. It is also an excellent approach to problems of hydrogeology, facilitates materials prospection and can guide designers in deciding what field reconnaissance and study is needed.

The many examples described illustrate the advantages of this approach.

An Example of Photogeology Applied to Small Scale Geotechnical Mapping, by R. Vyain.

This case history illustrates the use of photogeology in the geotechnical mapping of an equatorial zone. II made it possible to cover a large area to locate geotechnically complex areas requiring more thorough study.

The Arnon (France) Dam Foundation Problem, by J.Y. Scanvic.

Conventional aerial photography enables local fracture systems to be found without indicating their regional importance. Comparing such cove. rage with satellite scenes revealed a regional fault crossing the dam site, found during construction.

Seismotechnical Map of Provence at 1/250 000 Scale, by G. Weecksteen.

A basic seismotechnical map of the Provence area in France was drawn up from satellite scenes and aerial photographs. Problems arise in the methods to be used for handling this information. 


\section{Présentation de la séance $(*)$ \\ par Denis GALMIER \\ Ingénieur-Conseil}

L'interprétation des photographies aériennes et les autres techniques de télédétection présentent un intérêt pratique important, bien qu'encore mal ressenti, pour la géologie de l'ingénieur. Nous allons donc vous présenter un certain nombre d'applications concrètes et pratiques immédiatement utilisables. L'emploi de ces méthodes est susceptible d'améliorer sensiblement les résultats des recherches dans le domaine de la géologie de l'ingénieur en permettant de mieux situer les problèmes, de les intégrer dans l'environnement, et d'optimiser les recherches plus détaillées devant conduire à une meilleure sécurité des différents sites de génie civil.

L'application systématique de ces méthodes se traduit immédiatement par un gain important de compréhension, de précision et de qualité, ainsi que par une meilleure répartition des budgets d'étude, et une possible économie par non-augmentation du budget primitif.

Chronologiquement parlant, la photo-interprétation et la télédétection devraient intervenir systématiquement au début des programmes de recherches, en amont de toutes autres interventions techniques. Ceci est particulièrement valable pour les levers géologiques de terrain, qui doivent suivre et non précéder l'interprétation, étant entendu que l'examen des photographies aériennes sera repris en cours de lever et au niveau de la synthèse. Cette conviction a été acquise par les résultats obtenus à l'expérience de la pratique, dans tous les domaines d'application de la géologie.

Il est regrettable que, parfois, l'intervention de la photo-interprétation et de la télédétection soit demandée en cours de travaux, lorsque des ennuis n'ont pu être surmontés.

\section{A. - applications pratiques de la photo-interprétation et de la télédétection}

par Denis GALMIER, René LACOT, Raymond RICHARD

\section{INTRODUCTION}

Quelques exemples d'interprétation de photographies aériennes et de télédétection ont été choisis pour mettre l'accent sur certains types de phénomènes que ces techniques permettent de bien appréhender, ainsi que sur les modes de raisonnement et la logique des moyens employés pour obtenir les informations.

Ces exemples permettront de fournir une nouvelle approche des problèmes de sécurité du sol et du sous-sol, approche immédiatement utilisable à des fins pratiques.

Parmi les problèmes dont l'approche est ainsi réalisée, citons, tant en plaine qu'en montagne :

- les problèmes d'excavations: tassements, affaissements, karsts colmatés ou non ;

- fracturation et microfracturation;

- zones de rétention d'humidité ;

- anomalies morphologiques et micromorphologiques diverses ;

- ruptures de pente, risques de glissement de terrain, en petit ou en grand;

- approche des problèmes hydrogéologiques ;

- recherches de matériaux, graves, emprunts divers.

En montagne, glissements de terrain, étude de pentes, zones de chutes de pierres, etc.; les problèmes de géologie de l'ingénieur peuvent se trouver là plus aigus.
Pour autant que l'étude ponctuelle doive se faire, la vision de synthèse du site dans son environnement s'impose, en raison des interactions de l'un et de l'autre, et la photo-interprétation est une méthode préférentielle pour prendre de la « hauteur » et conduire à une saine réflexion.

L'interprétation se fait à des échelles d'images variant du 1/4000 (par exemple analyse de sites routiers très limités), au 1/1 000000 (ERTS, Landsat, Skylab); les premières pour des études très particulières avec pour résultat une bien meilleure organisation du budget; les secondes permettent parfois de faire apparaitre des éléments importants et inconnus autrement (par exemple découverte d'un chenal de sous-écoulement à fort débit dans une vallée, appuyé sur un linéament ERTS).

Chaque type de problèmes exigera une échelle et des documents particuliers. De toutes manières, il y a intérêt à exploiter ce qui existe déjà ; une couverture photographique d'un site en vue d'une photogrammétrie détaillée n'est pas forcément utilisable pour la photointerprétation. Il est aussi très utile de comparer les informations obtenues par plusieurs séries de photographies aériennes, ce qui permet d'apporter une appréciation du degré du risque et son évolution possible, donc un aspect dynamique.

(*) Séance du Comité français de Géologie de l’Ingénieur du 10 juin 1976. 


\section{EXEMPLES MONTRANT LE DEGRE DE FINESSE DE L'INTERPRETATION DANS LE DOMAINE DE LA DEFINITION DE LA CONSTRUCTIBILITE DE TERRAINS}

\subsection{Ville de M'Buji-Mayi, Kasaï Oriental, Zaïre}

L'exploitation du diamant est à l'origine de l'essor démographique exceptionnel de la ville qui atteint les 250000 habitants pour une surface bâtie de $40 \mathrm{~km}^{2}$ environ. Cette ville est établie sur un plateau couvert par d'épaisses formations argilo-sableuses (de 0 à $15 \mathrm{~m}$ ) surmontant des formations calcaréo-dolomitiques diverses de $250 \mathrm{~m}$ d'épaisseur reposant sur le socle.

La ville s'est développée assez harmonieusement jusqu'en 1960 (plan d'urbanisme, canalisation des eaux, boisement des zones non construites) : à partir de cette date le développement devient totalement anarchique, sans plan de voirie, avec un déboisement total des nouvelles zones urbanisées. Il se produit alors une érosion intense, notamment de la voirie non revêtue, l'accumulation de l'eau de pluie et des eaux usées dans les points bas non drainés, et l'enfoncement de plusieurs quartiers ou blocs d'immeubles.

Les questions qui furent posées en janvier 1971 étaient les suivantes :

a) Nature et extension des zones dangereuses en ville.

b) Ces zones existent-elles aussi à l'est de la ville où sont prévus des extensions?

L'étude par interprétation des photographies aériennes paraîssait la seule solution dans un premier temps car le tissu urbain ne permettait pas des campagnes de sondages ou une prospection géophysique. L'interprétation des photographies a couvert largement la banlieue de la ville (sur $400 \mathrm{~km}^{2}$ ) ; les photographies étaient à $1 / 30000$, prises quelques mois plus tôt. En étudiant la zone non urbanisée, on a pu confirmer qu'il s'agissait d'un phénomène de réactivation d'un karst. On a pu ensuite localiser très nettement les zones déprimées en forme de dolines dans les faubourgs; ces zones non perturbées par l'urbanisation étaient en équilibre. On a pu délimiter avec précision les zones effondrées et surtout les zones déprimées particulièrement sensibles, dans la ville.

Ces résultats figurent sur la carte présentée.

Les urbanistes savaient dès ce moment que :

- la ville pourrait s'étendre à l'Est, mais qu'elle serait soumise aux mêmes conditions géologiques que les les quartiers actuels;

- les constructions lourdes seraient particulièrement délicates à implanter ;

- la ville ne serait plus affectée par les effondrements que si des précautions impératives étaient prises.

Ces résultats furent naturellement vérifiés par la réalisation de campagnes de sondages et de géophysique bien préparées sur le canevas que la carte fournissait. Les résultats des études géotechniques ont confirmé et complété les premiers renseignements obtenus par photo-interprétation.

\subsection{Quartiers nord de la ville de Mâcon, Saône-et-Loire, France}

Cette petite étude a été réalisée pour illustrer un exposé fait aux responsables de l'aménagement de la région de Mâcon. La ville de Mâcon enserrée entre les Monts du Mâconnais et la Sâone se développe notamment vers le Nord sur un petit plateau constitué de séries jurassiques calcaires, recouvertes par les matériaux d'une haute terrasse.

Lors du début des travaux préparatoires à la construction d'un groupe d'immeubles collectifs, des désordres importants au niveau du sous-sol sont apparus, nécessitant le remaniement du plan de masse.

Il s'agissait notamment d'une «bétoire » ou doline située dans la zone même de construction et d'un certain nombre d'autres petites dépressions. Une étude géophysique relativement lourde fut faite qui permit de déterminer une zone non-aedificandi.

L'étude rapide des photographies aériennes au $1 / 20000$ a permis, en quelques heures, de localiser immédiatement et avec précision une série de petites dépressions bien marquées dans la morphologie et réparties sur le plateau. C'était là un paysage caractéristique d'un ancien karst ennoyé par des alluvions. Cet exemple montre que l'examen attentif au niveau de l'avant-projet des photographies aériennes, aurait $\mathrm{pu}$, pour une dépense minime par rapport au coût total de l'opération, orienter l'architecte et éviter des pertes de temps et d'argent.

\subsection{Saint-Witz (Val d'Oise)}

Le domaine à urbaniser, d'une surface d'environ 60 ha, se situe sur le flanc sud-ouest de la butte de Montmélian (fig. 1 et 2 ).

L'étude a consisté à étudier l'environnement du site en particulier dans son aspect géomorphologique et l'aptitude à la fondation.

La complication est plus grande, et pour approcher les problèmes d'une telle étude, sept couvertures aériennes différentes ont été utilisées s'échelonnant de mai 1955 à août 1972, d'échelle variant entre le $1 / 15000$ et le $1 / 30000$.

La série stratigraphique intéressant le domaine est la suivante :

- dépôts superficiels : colluvions polygéniques et limons des plateaux recouvrant :

- sables de Fontainebleau ;

- marnes vertes;

- en profondeur, marnes supragypseuses et gypse anciennement exploité entre Plailly et Saint-Witz (La Carrière, La Boulaie), en amont pendage, le domaine étant situé sur le flanc sud-ouest d'une structure anticlinale.

Cinq types de phénomènes ont été observés sur les photographies aériennes, avec, en plus, quelques traces tectoniques :

- des rétentions d'humidité en larges taches;

- des points singuliers, quasiment ponctuels, d'humidité et d'infiltration, présentant un caractère plus ou moins répétitif, d'un à quelques mètres carrés ;

- des affaissements en grand (diamètre $100 \mathrm{~m}$ et au-delà), dus à la dissolution du gypse en profondeur ;

- des risques de glissement de terrain provoqués par le déplacement de lames allongées immédiatement au sud-est de Saint-Witz ;

- enfin des phénomènes de foisonnement dans la zone des marnes vertes. 


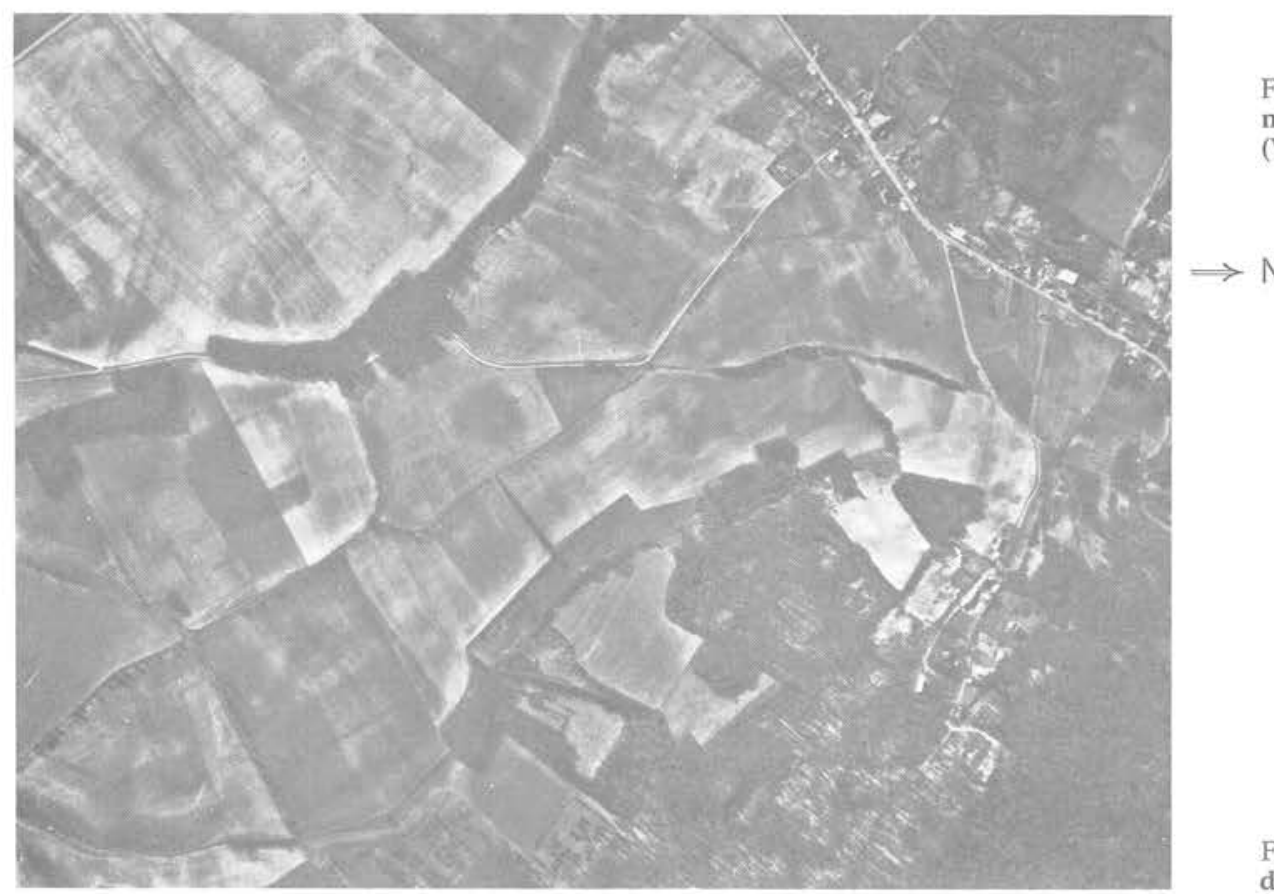

Fig. 2. - Résultat de l'étude du domaine de Saint-Witz.
Fig. 1. - Vue aérienne du domaine à urbaniser de St-Witz (Val-d'Oise).
Les principales constatations retenues sont les suivantes :

a) l'évolution de la forme et de la surface des taches de rétention d'humidité permet de définir les secteurs sensibles avec forte imbibition superficielle;

b) la répétitivité des points singuliers (certains observés trois fois, un cinq fois), constitue un danger très localisé, qui a été contrôlé et vérifié par les études sur le terrain;

c) l'intérieur des dépressions en grand est évidemment à éviter pour la construction;

d) l'analyse des phénomènes de déplacement de lames superficielles et de foisonnement doit être confirmée par les méthodes appropriées.

Ce domaine présente des conditions d'environnement (accès, climat, ensoleillement, etc.) extrêmement favorables à la réalisation d'un ensemble pavillonnaire, et il restera au promoteur le mérite d'avoir fait utiliser judicieusement l'ensemble des techniques pouvant lui assurer la sécurité.

Le contrôle de terrain, exécuté le 22 décembre 1972 , par temps de gel superficiel, avec l'appui d'une tarière à main, a confirmé l'exactitude des faits observés. En particulier, la tache ponctuelle quintuple a révélé l'eau à $1.50 \mathrm{~m}$ de profondeur, alors qu'autour, le terrain reste parfaitement $\mathrm{sec}$; la tenue des limons y est très médiocre.

Dans la tache d'humidité diffuse située à $100 \mathrm{~m}$ au sud du point quintuple, la tarière a révélé des limons argilo-sableux plus compacts à veinules brunes et noires, et l'eau à $2.50 \mathrm{~m}$.

Deux tests à la tarière exécutés sur la pente sud-est de Saint-Witz ont montré, l'un des colluvions plus sableuses recouvrant les sables de Fontainebleau avec l'eau à $2.20 \mathrm{~m}$ après quelques minutes, l'autre des colluvions argileuses sur substratum de marnes avec l'eau à $1.40 \mathrm{~m}$. Ces deux tests démontrent la fragilité superficielle de la pente près de Saint-Witz.

Le contrôle de terrain a donc parfaitement confirmé les données de la photo-interprétation.

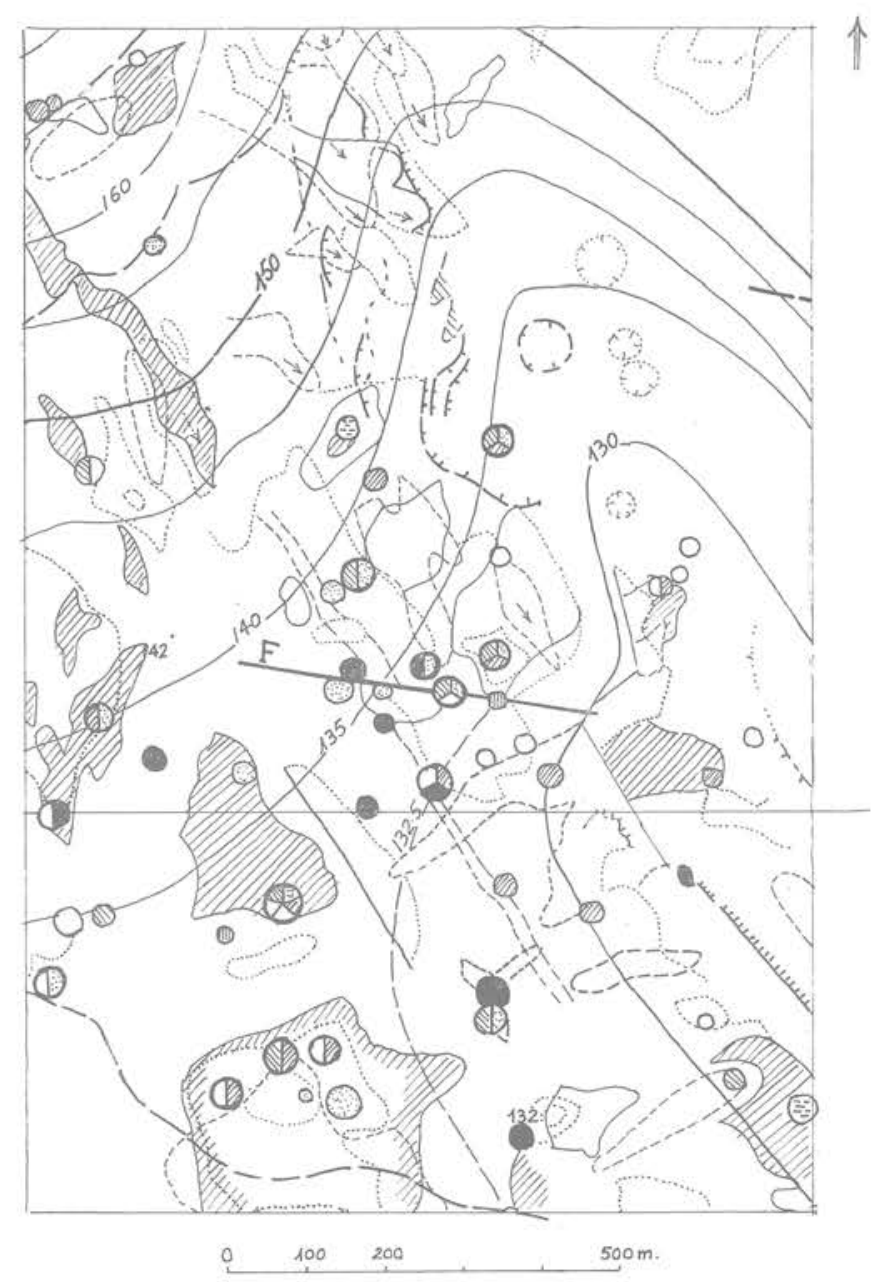

Evidemment, un programme de recherches géotechniques a suivi, mais il a pris en compte, et c'est très important, toutes ces informations. 


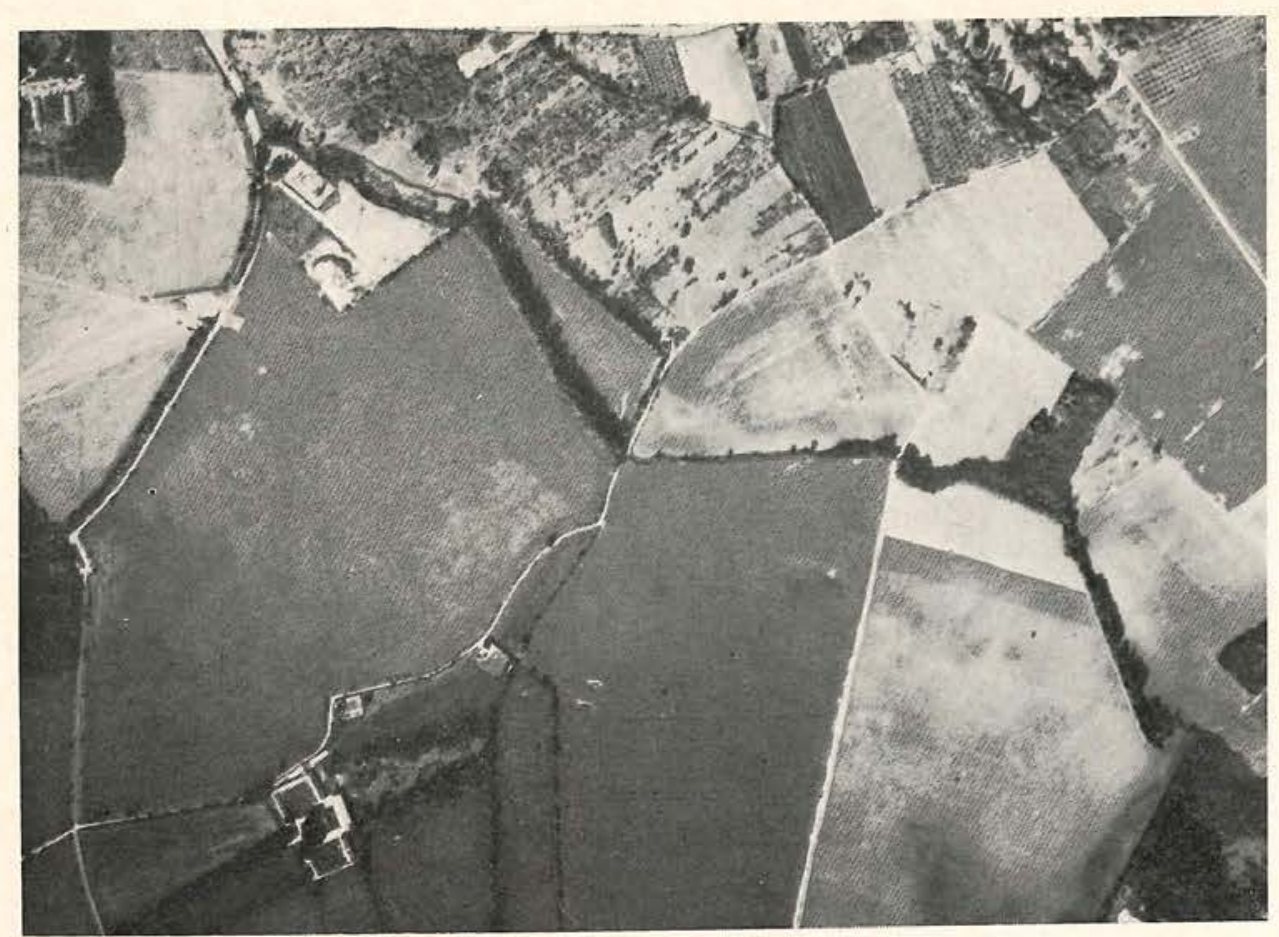

Fig. 3. - Vue aérienne du domaine à urbaniser des Coteaux de Maubué (Ville Nouvelle de Marne-la-Vallée).

\subsection{Marne-la-Vallée - Les Côteaux de Maubué (Seine-et-Marne)}

Dans le secteur 2 de la Ville Nouvelle de Marnela-Vallée, le domaine des Côteaux de Maubué doit être équipé pour recevoir plus de 1000 logements. Dans le cadre d'une étude géotechnique, l'intervention de la photo-interprétation a été demandée pour rechercher les glissements ou amorces de glissement sur chaque flanc du vallon du Ru-de-Maubué qui auraient pu nuire à la constructibilité du site (fig. 3).

Le terrain est bouleversé depuis 1974 , et seules les photographies aériennes de 1972 et antérieures ont pu reconstituer la surface du sol.

Six séries de photographies aériennes ont été interprétées, et l'ensemble a fait l'objet d'une carte au $1 / 5000$ en deux volets :

- un document de photo-interprétation ;

- une carte géologique améliorée au 1/5000.

Le substratum du domaine est constitué essentiellement sous les limons des plateaux, par la formation de Brie (argiles, meulières), les marnes vertes et glaises à Cyrènes et les marnes blanches de Pantin et les marnes bleues d'Argenteuil. Le vallon est dissymétrique, avec flanc nord-est à forte pente, flanc sud-ouest adouci ; le vallon suit une inflexion structurale entre le flanc nord d'un anticlinal et un repli périclinal latéral au sud de Torcy. Ceci explique la raideur du flanc nord-est du vallon.

L'interprétation a mis en évidence des phénomènes ponctuels :

- des points singuliers parfois répétitifs (doubles ou triples) suspects ;

- des " centres d'infiltration » avec un point sombre au centre et un anneau périphérique clair, indiquant une microaire de drainage, ou éventuellement une ancienne marnière ;

et des phénomènes plus étendus, à savoir :

- des surfaces sombres répétitives (parfois trois ou quatre fois), à rétention d'humidité superficielle, à surveiller ;
- des surfaces claires répétitives, correspondant à des marnes sub-affleurantes ou à des recouvrements perméables ;

- des dépressions bien localisées, la plus remarquable se trouvant au Sud de la carte et à l'extérieur du domaine, soulignée par un essaim de points singuliers groupés.

Les dépressions peu marquées peuvent correspondre à des tassements, les dépressions bien marquées ont probablement une signification hydrogéologique.

La photo-interprétation permet également d'analyser, spécialement sur le flanc nord-est du vallon, les glissements et amorces de glissement. Les mêmes différenciations entre lames suivant la pente et bandes d'amorce de glissement observées sur Saint-Witz sont retrouvées ici, le degré de risque est présumé par la répétitivité de l'observation.

Les paquets glissés sont également identifiés, marqués par des mélanges de sol visibles sur les photographies aériennes; ceux qui risquent de glisser sont aussi repérés. Une surveillance est donc possible sur les zones les plus instables.

L'échelle des photographies (du 1/15 000 au 1/30 000) suffit, lorsque les contrastes sont bien marqués, pour dégager toutes ces observations. Elles ont été portées ensuite sur une carte au $1 / 5000$.

\subsection{La Chapelle-Saint-Mesmin (Loiret)}

Ce petit domaine (4 ha environ) est pratiquement plat, avec substratum de calcaire de Beauce, sans autre précision. La carte a été établie à l'échelle du $1 / 2000$

Cinq séries de photographies aériennes panchromatiques ont été exploitées (1955 à 1973), ainsi qu'une image couleurs d'août 1973.

Les phénomènes suivants ont été observés :

- des taches sombres pouvant correspondre à des rétentions superficielles d'humidité, taches qui ont tendance à se grouper surtout au sud-ouest et à l'est du domaine : la répétitivité atteint 3 , parfois 4 ; 
- une possible anomalie structurale correspondant à une zone un peu plus haute ;

- des « centres d'infiltration », situés à l'extérieur du domaine ;

- des alignements qui, correspondant à la direction tectonique régionale, sont probablement des bandes fracturées (Nord 100 à Nord $115^{\circ}$ Est).

L'intégration du plan-masse réduit au 1/2000 dans la carte permet immédiatement de définir les secteurs où les essais géotechniques devront être approfondis :

- au Sud, l'un des deux immeubles prévus sur le planmasse ;

- à l'Est, deux groupes totalisant sept logements.

Il y a évidemment d'autres secteurs à surveiller.

Cette étude montre que, même sur une formation monotone à petite échelle, des différenciations peuvent apparaître et que l'image photographique est un remarquable moyen d'approche, qui permet d'obtenir une synthèse et de préciser les secteurs sensibles.

\subsection{Domaine de Béthanie, à Montsoult (Val-d'Oise)}

Ce tout petit domaine (4 à 5 ha environ) est situé au sud-ouest du village de Montsoult, sur la pente sud d'une colline de sables de Fontainebleau surmontés par les meulières de Montmorency (Le Parc).

L'interprétation a consisté à examiner les éventuels problèmes qui se posaient.

Les formations géologiques intéressant le domaine sont les suivantes de haut en bas :

- sables et grès de Fontainebleau ;

- marnes à huîtres ;

- calcaire de Sannois, très mince, 2 m environ ;

- marnes vertes, glaises à Cyrènes ;

- marnes supragypseuses dans la partie sud, en contrebas.
Le plan-masse avait été établi antérieurement au $1 / 500$. Le domaine est assez proche d'anciennes exploitations de gypse (Le Clos Mangeot), dont certaines souterraines.

L'interprétation post-contrôlée sur le terrain a révélé des problèmes hydrogéologiques et des problèmes géotechniques.

\section{Hydrogéologie}

Le domaine est traversé par deux thalwegs principaux, très mouillés, l'origine de l'eau se situant à la base des meulières de Montmorency.

Par ailleurs, deux zones d'humidité principales, bien marquées sur le terrain par une végétation hydrophile, correspondent à des exutoires de nappes aux niveaux 140-145 m. Le tracé de ces informations, d'abord au $1 / 5000$, puis au $1 / 500$, situe immédiatement les pavillons qui risquent d'être atteints.

\section{Géotechnique}

Trois types de problèmes ont été mis en évidence : - poussées sur les murs de soutènement, en cours de déformation;

- phénomènes de tassement ou d'affaissement localisés, à contours circulaires ou ovales, en relation possible avec des écoulements souterrains ;

- des risques de glissement en masse, les terrassements risquant de remettre des lames superficielles en mouvement.

Une campagne de sondages à la tarière a confirmé l'exactitude des informations fournies par la photointerprétation.

Il ne semble pas y avoir de risques d'affaissements profonds dus au gypse.

2.7. Les six exemples montrent le degré de finesse atteint par l'interprétation des photographies aériennes. Mais elle nécessite une grande expérience basée sur des qualités d'observations et de synthèse.

\section{EXEMPLES D'INTERPRETATION DES ZONES A FORT RELIEF, DES PROBLEMES HYDROGEOLOGIQUES ET DES RECHERCHES DE MATERIAUX D'EMPRUNT}

\subsection{Le Chaînon de Vuache (Haute-Savoie)}

Ce chaînon, qui diverge de l'ensemble des Monts du Jura, et que traverse le Rhône au Défilé de l'Ecluse, doit être traversé en tunnel par le tracé de l'autoroute A 42 Lyon-Genève entre Arcine et Dingy-en-Vuache.

L'interprétation a permis de rechercher les points sensibles du tracé dans la traversée du chaînon (fig. 4 et 5).

Les formations géologiques suivantes sont rencontrées par le futur tunnel; du Sud-Ouest au Nord-Est; elles s'inclinent vers le nord-est :

- Le Portlandien à calcaires dolomitiques avec cargneules.

- Le Purbeckien, épais de quelques mètres seulement sous le relief sommital du chaînon.

- Le Valanginien, marqué par un ressaut situé légèrement à l'Ouest de la ligne de crête, essentiellement calcaire avec des passées marneuses.

- L'Hauterivien qui occupe le flanc oriental, avec une morphologie plus douce, est constitué de calcaires marneux et marnes.
Ces étages ayant été relevés sur la carte géologique Nantua au 1/80 000, l'interprétation a permis de préciser les contours avec une précision très supérieure à celle du $1 / 25000$.

Mais en outre, elle a posé les problèmes de géologie de l'ingénieur auxquels sera confrontée la construction du tunnel, les principaux étant :

a) Des réseaux de failles et fractures, particulièrement denses dans le secteur du tracé, avec une direction dominante Nord $110^{\circ}$ Est et Nord-Sud subordonnée, la principale se plaçant dans le Portlandien, à environ $250 \mathrm{~m}$ de l'entrée occidentale du tunnel.

b) des karsts, plus particulièrement concentrés dans les formations valanginiennes, et formant un essaim très dense sur une tranche de $500 \mathrm{~m}$ du futur tracé.

Dans ce cas, comme d'habitude, l'interprétation a été étendue sur une bande assez large de part et d'autre pour permettre d'intégrer ces observations dans leur environnement. Nous pouvons ainsi prendre du recul et émettre une opinion objective et correcte sur la géologie du secteur. 
Fig. 4. - Vue aérienne du châ̂non de Vuache (Haute-Savoie).

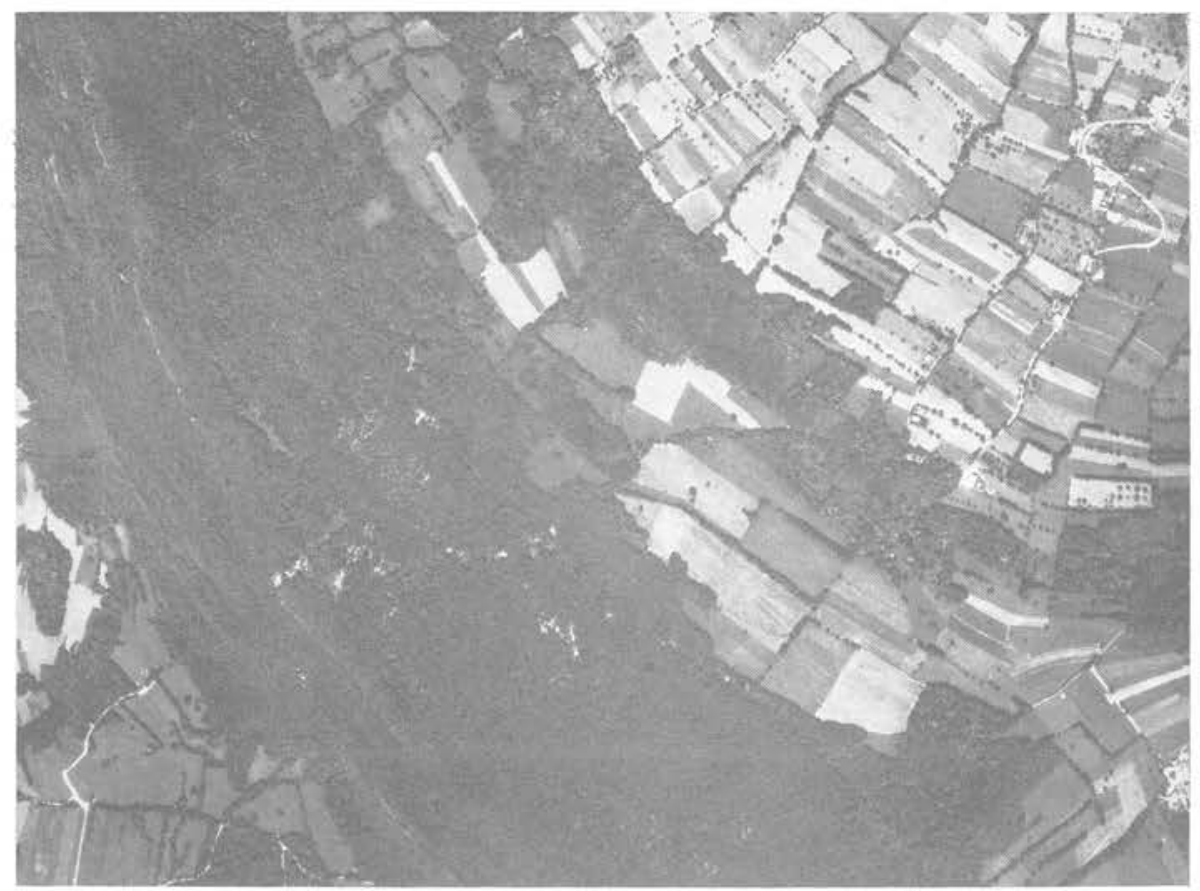

\subsection{Exemple de la Vallée du Doubs, au sud de Dôle (Jura)}

Un exemple intéressant d'interprétation de photographies aériennes est celui de la vallée du Doubs, en aval de son confluent avec la Loue, où a été réalisée une recherche hydrogéologique qui présente également un intérêt pour la géologie de l'ingénieur.

L'interprétation a utilisé les informations fournies par l'image ERTS $1 \mathrm{n}^{\circ} 1078$ - 09553 du 9 octobre 1972 et une série de photographies aériennes panchromatiques et infra-rouge noir et blanc au 1/15000.

Les résultats ont fait l'objet d'une carte au 1/25000.

Dans la vallée et à proximité, l'image ERTS et les photographies ont révélé l'importance d'un réseau d'accidents orientés Nord $30^{\circ}$ Est.
Par ailleurs, nous avons pu séparer dans la vallée des traînées plus ou moins contournées, ayant une micromorphologie très particulière, les unes claires, les autres sombres, et qui correspondent à des différenciations de la perméabilité et également par conséquent à des différences lithologiques (fig. 6).

Parmi les traînées claires, l'une est très remarquable, orientée Nord $50^{\circ}$ Est, elle est constituée d'une succession de petites taches sur plus de $3 \mathrm{~km}$. C'est là, entre le Doubs et Chaussin, qu'a été découvert un chenal de sous-écoulement profond de $14.50 \mathrm{~m}$, aquifère sur $11 \mathrm{~m}$, présentant des galets de la grosseur du poing, avec une excellente perméabilité.

Ce résultat hydrogéologique positif ne doit pas faire perdre de vue l'intérêt de ces techniques pour la recherche de graves, et matériaux d'emprunt divers, puisqu'elle fournit en même temps des indications sur la localisation probable des matériaux recherchés.

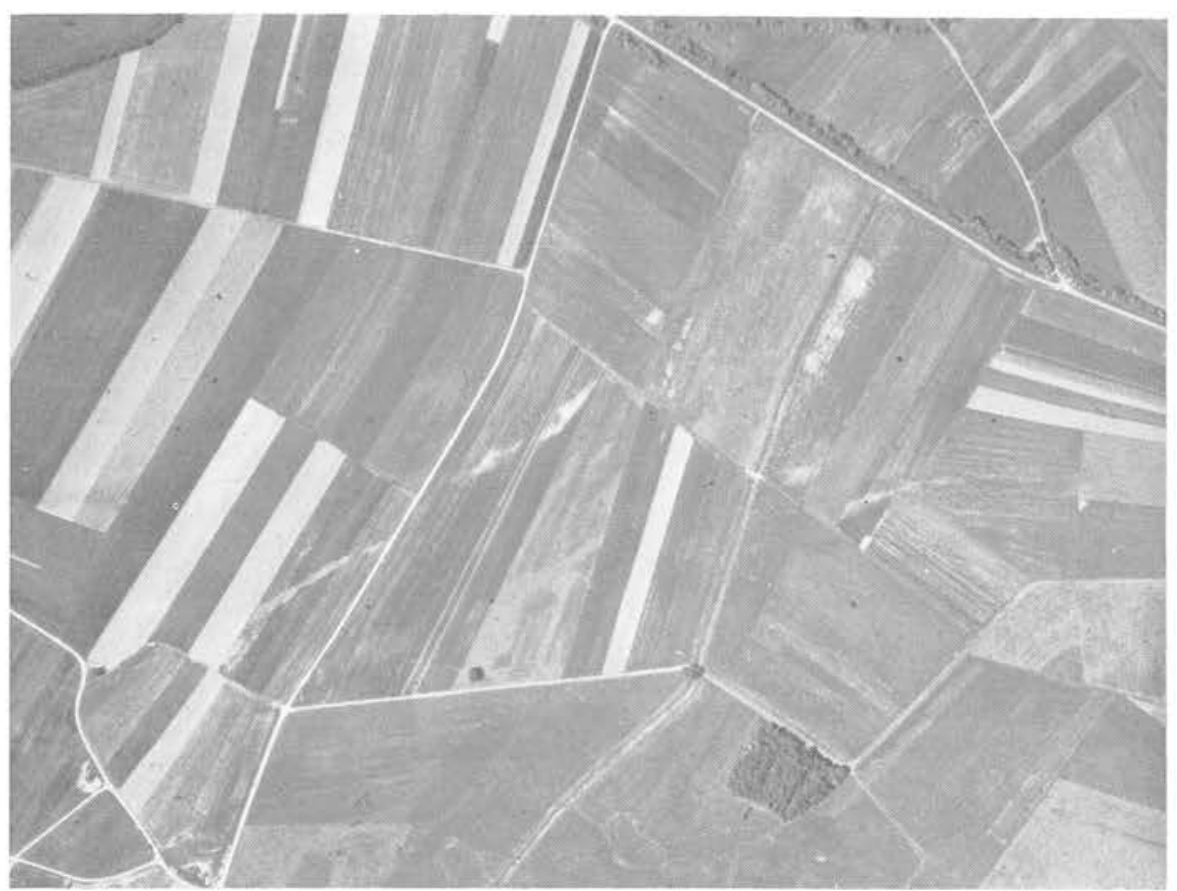

Fig. 6. - Vue aérienne de la Vallée du Doubs. 


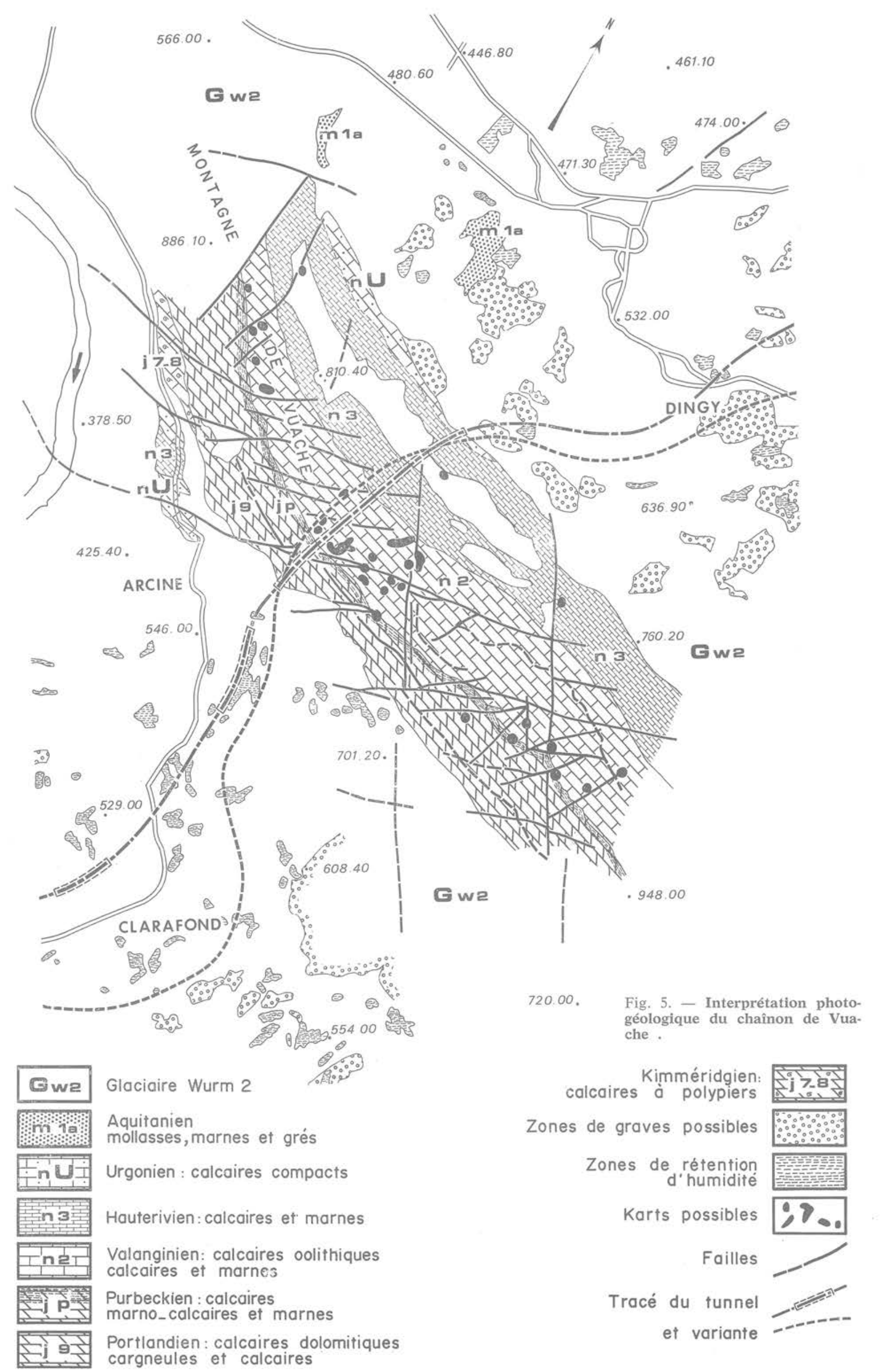




\section{CONCLUSIONS}

Les exemples cités apportent les renseignements suivants :

1) les techniques de photo-interprétation et télédétection sont à appliquer en amont de toutes les études concernant la géologie de l'ingénieur ;

2) ces techniques permettent la compréhension des relations entre le site et son environnement, donc une bonne synthèse ;

3) elles permettent de définir la nature des risques et parfois le degré de risque ;

4) elles présentent une souplesse d'utilisation, en raison des possibilités d'adaptation des documents à chaque type de problèmes ;

5) elles engagent des moyens matériels extrêmement modestes, les interventions sont rapides et discrètes ;

6) par une collaboration pluridisciplinaire avec géologues de terrain, géotechniciens, géophysiciens, elles permettent de cerner l'ensemble des problèmes, d'expliquer plus rapidement des anomalies ressenties par une autre discipline, et d'orienter les travaux vers les zones les plus sensibles.

Naturellement, ces mêmes enseignements sont applicables à des domaines très variés, comme le prouvent les résultats concrets que nous avons obtenus en géotechnique, hydrogéologie, recherche minière, aménagement, etc.

Il est certain que ces techniques ont un très grand intérêt pour l'hydrogéologie et pour les études préliminaires d'aménagement du territoire (analyse des paysages, et des différents tissus naturels et artificiels), d'environnement, pour la préparation des SDAU, des POS, etc.

Il s'agit donc finalement d'un très vaste domaine, dont toutes les possibilités sont loin d'avoir été entièrement explorées.

\section{B. - exemple d'utilisation de la photogéologie en cartographie géotechnique à petite échelle}

\section{par René VYAIN}

Une carte géotechnique à l'échelle du 1/500 000 était prévue dans une région d'Afrique équatoriale couverte aux deux tiers par la forêt et au tiers par la savane. Elle avait pour but de mettre en évidence les principales contraintes naturelles pouvant influencer l'aménagement de cette province dans les domaines routiers, urbains, agricoles et industriels.

On disposait d'une couverture cartographique uniquement planimétrique et d'une esquisse géologique peu fiable.

Etant donné l'étendue de l'étude $\left(35000 \mathrm{~km}^{2}\right)$, les essais géotechniques devaient être répartis judicieusement afin qu'un minimum de mesures reste significatif de l'ensemble.

Aussi, une étude photogéologique a-t-elle été entreprise afin de servir d'instrument de travail préliminaire à l'établissement de cette carte. Une couverture aérienne à $1 / 40000$ a été utilisée pour établir une carte géomorphologique à $1 / 200000$. Cette carte tendait à définir des ensembles homogènes à l'intérieur desquels on pouvait penser que les contraintes géotechniques seraient comparables.

L'étude au sol par les équipes géotechniques n'ayant pas encore été entreprise, il n'est pas possible actuellement de préciser la correspondance entre unités photogéologiques et géotechniques.

Cependant, il est possible de donner une estimation de l'aptitude de la photogéologie à définir des ensembles homogènes à cette échelle du $1 / 200000$. Basés sur des critères de pente, d'affleurement, de réseau hydrographique, d'inondabilité, de végétation, de structure et de tectonique, la cartographie a permis de distinguer une vingtaine de faciès différents dont la surface cumulée couvre environ $90 \%$ du secteur. Quelques profils judicieusement placés devraient suffire dans la majorité des cas à en définir les caractères géotechniques. Les $10 \%$ restant sont des zones à morphologie ou à structure complexe qu'il est difficile de cartographier à cette échelle. Ils ont été simplement délimités. Il est d'ailleurs probable que ces zones complexes soient également rebelles à une analyse géotechnique à petite échelle.

A l'échelle utilisée, la préparation photogéologique semble bien adaptée dans des ensembles géologiques à structure monotone ou répétitive. Elle a l'avantage également de permettre de localiser les zones nécessitant une échelle de cartographie plus grande. 


\section{C. - problème posé par la fondation du barrage de l'arnon (cher)}

par Jean-Yves SCANVIC

Cette note présente les résultats d'une étude comparée de photo-fracturation réalisée à partir de photographies aériennes et d'images de satellite.

Situé dans le département du Cher (France), le barrage de l'Arnon est un barrage poids en béton d'une hauteur totale de $29 \mathrm{~m}$. Il est destiné à la desserte en eau du syndicat inter-communal, à régulariser le débit de l'Arnon et à participer à l'aménagement touristique de la région.

Les études géologiques et géotechniques ont été réalisées par le BRGM. La division photogéologie et télédétection a été chargée d'une analyse de la fracturation sur les documents de télédétection existants, photographies aériennes panchromatiques à $1 / 25000$ de l'IGN et images ERTS-1 à 1 million de la NASA.

L'étude des photographies aériennes a montré (fig. 1) l'existence de quatre familles de failles $\mathrm{N} 170^{\circ}$ est (type 1) $\mathrm{N} 20^{\circ}$ est (type 2) $\mathrm{N} 70^{\circ}$ est (type 3) nord $110^{\circ}$ (type 4). Aucune de ces failles ne semblait avoir d'importance réelle et les premières observations sur le terrain le confirmaient.

L'étude non stéréoscopique des images orbitales ERTS sur la bande spectrale $(0.8$ à $1.1 \mu)$ (fig. 2$)$ a permis de repérer avec une précision satisfaisante le site retenu pour le barrage et a attiré l'atiention sur l'existence de trois accidents d'importance régionale, l'un pouvant passer dans l'axe de la vallée ( $\mathrm{n}^{\circ} 2$ sur la figure), le second recoupant la vallée un peu en aval du barrage (4), le dernier enfin étant oblique au précédent (3).

L'ensemble des investigations géologiques ultérieures a mis en évidence l'existence et l'importance de la faille de type 2 (longitudinale) et de la faille du type 4 (transversale à pendage amont) au niveau du site du barrage : cette dernière, il convient de le souligner, n'a pu être mise en évidence, malgré son importance (remplissage argileux de $2 \mathrm{~m}$ d'épaisseur) qu'après les premiers travaux de décapage du site.

Fig. 1. - Barrage de l'Arnon (Cher).

Interprétation des photographies aé-

riennes à $1 / 30000$.

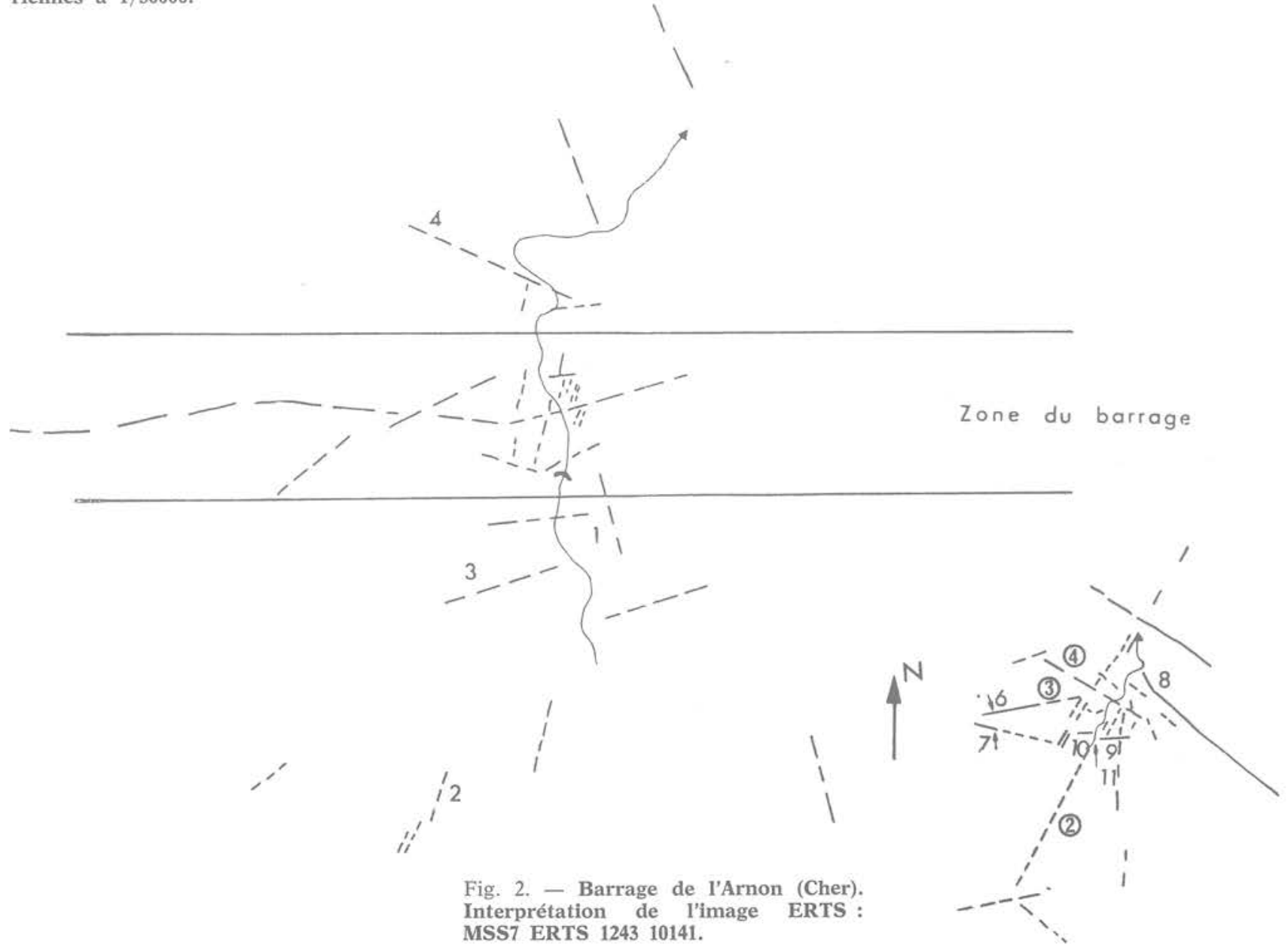




\section{D. - esquisse sismo-tectonique de provence à $1 / 250000$}

par Guy WEECKSTEEN

Dans le cadre de l'élaboration d'une esquisse sismotectonique de la Provence, prélude à la mise au point d'une carte sismo-tectonique de la France, en complément des données de séismicité historique, de néotectonique, de géophysique, les données de télédétection spatiale ont été utilisées et ont permis un recensement des linéaments.

Ce recensement est fondé sur l'exploitation de :

- photographies et images spatiales de la NASA: Skylab et Landsat 1;

- photographies aériennes à 1/100000 de l'Institut géographique national pour une partie de la carte.

C'est de manière délibérée que dans la phase d'interprétation, ce travail a été réalisé dans l'esprit d'un inventaire strictement analytique.

Pour cette raison, on s'est interdit toute généralisation et toute confrontation avec le contexte géologique structural.

La confrontation entre les différents documents analytiques n'intervient qu'au stade de l'établissement de la carte de synthèse.

Parmi les problèmes de méthode rappelons :

- que les documents spatiaux, s'ils fournissent une vision synoptique permettant d'intégrer des éléments linéaires discontinus et de nature variée, ils ne procurent pas la stéréoscopie, ce qui implique une hiérarchisation à l'aide par exemple de photographies aériennes à petite échelle ;

- que le système d'acquisition des données par balayage (Landsat) introduit un lignage artificiel orienté $\mathrm{N} 100^{\circ}$ et que le filtrage de cette direction par l'interprétateur peut l'avoir amené à minimiser les linéaments de même direction. 